\title{
Clostridium difficile Infection in Patients With Chronic Kidney Disease
}

\author{
Mira T. Keddis, MD; Sahil Khanna, MBBS; Amit Noheria, MBBS; Larry M. Baddour, MD; \\ Darrell S. Pardi, MD, MS; and Qi Qian, MD
}

\begin{abstract}
Objective: To examine the rate of Clostridium difficile infection (CDI) and hospital-associated outcomes in a national cohort of hospitalized patients with chronic kidney disease (CKD) and assess the impact of long-term dialysis on outcome in these patients.

Patients and Methods: Data for January 1, 2005, through December 31, 2009 were obtained from the National Hospital Discharge Survey, which includes information on patient demographics, diagnoses, procedures, and discharge types. Data collected and analyzed for this study included age, sex, race, admission type (urgent or emergent combined vs elective), any colectomy diagnosis, length of stay, type of discharge, and mortality. International Classification of Diseases, Ninth Revision, Clinical Modification codes were utilized to identify CKD patients and CDI events. Weighted analysis was performed using JMP version 9. Results: An estimated 162 million adults were hospitalized during 2005-2009, and 8.03 million (5\%) had CKD (median age, 71 years). The CDI rate in CKD patients was $1.49 \%$ ( 0.119 million) compared with $0.70 \%$ ( 1.14 million) in patients without $C K D(P<.001)$. Patients with $C K D$ who were undergoing long-term dialysis were more than 2 times as likely to develop CDI than non-CKD patients and 1.33 times more likely than CKD patients not undergoing dialysis (all $P<.001$ ). In a weighted multivariate analysis adjusting for sex and comorbidities, patients with CKD and CDI had longer hospitalization, higher colectomy rate (adjusted odds ratio [aOR], 2.30; 95\% confidence interval [CI], 2.14-2.47), dismissal to a health care facility (aOR, 2.22; 95\% CI, 2.19-2.25), and increased in-hospital mortality (aOR, 1.55; 95\% CI, 1.52-1.59; all P<.001) as compared with CKD patients without CDI. Patients with CKD who were undergoing long-term dialysis did not have worse outcomes as compared with CKD patients who were not undergoing long-term dialysis.

Conclusion: These data suggest that patients with CKD have a higher risk of CDI and increased hospital-associated morbidity and mortality. Future prospective studies are needed to confirm these findings and to identify effective CDI prevention in CKD patients, who appear to have an increased risk of CDI acquisition.
\end{abstract}

(C) 2012 Mayo Foundation for Medical Education and Research — Mayo Clin Proc. 2012;87(11):1046-1053

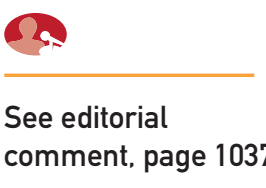

From the Division of $\mathrm{Ne}$ phrology and Hypertension (M.T.K., Q.Q.), Division of Gastroenterology and Hepatology (S.K., D.S.P.), and Division of Infectious Diseases (L.M.B.), Mayo Clinic, Rochester, MN; and Division of Cardiology, Cedars-Sinai Medical Center, Los Angeles, CA (A.N.)
C lostridium difficile infection (CDI) is the most common cause of transmissible nosocomial infection in health care facilities ${ }^{1,2}$ and is associated with increasingly significant morbidity and mortality. ${ }^{3-5}$ The clinical presentation of CDI spans the spectrum from asymptomatic colonization to fulminant pseudomembranous colitis, septic shock, and death. ${ }^{6}$ Known risk factors for CDI include antibiotic exposure, ${ }^{7}$ older age, ${ }^{3,8}$ gastric acid suppression therapy, ${ }^{9,10}$ immunosuppression, ${ }^{11}$ and prolonged hospitalizations. ${ }^{12}$ Patients with kidney disease are at heightened risk for many of these known risk factors for CDI and therefore represent a vulnerable group of patients at increased risk of CDI and CDI-associated morbidities. In fact, the Infectious Diseases Society of America has recognized acute kidney injury (AKI) as a marker of severe CDI. ${ }^{2}$ However, data on patients with chronic kidney disease (CKD) and associated risk and outcomes of CDI are scarce, are limited by small sample size, and have generated inconsistent results. For example, some studies advocated that CDI prevalence is greater among all patients with renal disease compared with those without renal disease, ${ }^{13}$ whereas others concluded that only patients undergoing dialysis are at highest risk of CDI. ${ }^{14,15}$ Similarly, none have evaluated whether long-term dialysis modifies the outcomes of CDI in CKD patients. We hypothesized that the rate of CDI is increased in patients with CKD and is associated with worse morbidity and mortality, especially among patients undergoing long-term dialysis. Our objectives were to (1) examine the rate of CDI in a large cohort of hospitalized CKD patients compared with a control group of patients without CKD, (2) evaluate hospital-associated morbidity and mortality in CKD patients with and without CDI, and (3) assess the impact of longterm dialysis on CDI outcomes using a large national database of hospitalized patients.

\section{PATIENTS AND METHODS}

\section{Data Source}

The National Hospital Discharge Survey (NHDS) is a national survey that has been conducted annually since 1965. It collects hospital discharge information from nonfederal short-stay hospitals (defined as 
average length of stay of $<30$ days) throughout the United States via a stratified random sampling process. The database contains patients' diagnoses and procedure codes, demographic characteristics, type of admission, length of stay, hospital mortality, and type of dismissal (eg, to home or to a health care facility). The database is publically available online at http://www. cdc.gov/nchs/nhds.htm, and 2009 data were released in April 2011. Diagnoses are based on the International Classification of Diseases, Ninth Revision, Clinical Modification (ICD-9-CM) codes.

\section{Data Collection}

Data collected and analyzed for this study included age, sex, race, admission type (urgent or emergent combined vs elective), any colectomy diagnosis, length of stay, type of discharge, and mortality for all patients discharged between January 1, 2005, through December 31, 2009. Patients younger than 18 years were excluded. The type of hospital discharge is classified by the NHDS database into the following categories: routine and/or discharged home, discharged to a short-term health care facility, discharged to a long-term health care facility, unknown discharge status, left against medical advice, or death during hospitalization. In our study, we referred to death during hospitalization as inhospital mortality and analyzed this as a separate clinical outcome. We also combined patients discharged to short- and long-term health care facilities and compared them with patients who had a routine and/or home dismissal.

The ICD-9-CM codes were utilized to identify CKD patients and CDI events. For patients with CKD, the ICD-9-CM code corresponding to unspecified CKD (585.9) and CKD stages 1 to 5 (CKD- 1 to CKD-5) (585.1-585.6) was identified. For patients with CKD-5, code 585.5 corresponds to those with CKD-5 who are not undergoing dialysis, whereas code 585.6 corresponds to CKD-5 patients who are undergoing dialysis. For analysis of overall CDI occurrence rate and CDI-associated outcomes, all CKD stages (including unspecified CKD) were included. The ICD-9-CM codes for CKD stages are based on the National Kidney Foundation Kidney Disease Outcome Quality Initiative Guidelines of estimated glomerular filtration rate (eGFR) (CKD-1, $\geq 90 \mathrm{~mL} / \mathrm{min}$ per $1.73 \mathrm{~m}^{2}$; CKD-2, 60-89 mL/min per $1.73 \mathrm{~m}^{2}$; CKD-3, 30-59 mL/min per $1.73 \mathrm{~m}^{2}$; CKD-4, 15-29 mL/min per $1.73 \mathrm{~m}^{2}$; and CKD-5, $<15 \mathrm{~mL} / \mathrm{min}$ per $1.73 \mathrm{~m}^{2}$ or undergoing dialysis). ${ }^{16}$ $C$ difficile infection was defined by a unique ICD9-CM code, 008.45. We also collected information on any diagnosis of AKI using 6 unique ICD-9-CM codes $(584,584.5,584.6,584.7,584.8$, or 584.9) and on whether any form of renal replacement therapy was administered during the hospitalization us- ing Current Procedural Terminology codes for hemodialysis and peritoneal dialysis.

Comorbid conditions were collected from the database using the Healthcare Cost and Utilization Project guidelines (http://www.hcup-us.ahrq.gov/ toolssoftware/comorbidity/comorbidity.jsp). Clinically relevant comorbid conditions were abstracted and used for statistical adjustment. These conditions included cardiovascular disease, which was defined as congestive heart failure, valvular disease, and pulmonary and circulatory diseases; peripheral vascular disease; diabetes mellitus and associated complications; hypertension-associated complications; liver diseases; and obesity.

\section{Statistical Analyses}

The summary database was converted to a JMP file version 9.0.1 (SAS Institute, Cary, NC) using SAS version 9.2. Demographic and clinical outcome data were analyzed using the $t$ test for normally distributed continuous variables and the Wilcoxon rank sum test if not normally distributed (including age and length of stay). The $\chi^{2}$ test of independence was used to compare binary variables. For comparison of continuous data among several groups, analysis of variance was used if the data were normally distributed, and the Kruskal-Wallis test was used if data were skewed. Results of continuous variables are reported as mean and median (minimum, maximum) when appropriate. Comparisons of categorical variables are reported as percentage, odds ratios (ORs), and 95\% confidence intervals (CIs). Multivariate linear and logistic regression models with weighted analysis were used to adjust for the effect of sex and comorbid conditions on CDIassociated outcomes. We did not include age in the multivariate models because CKD stage assignment is based on eGFR, which includes age in the estimate equation. Weighted analysis was performed in order to obtain nationwide estimates and account for the stratified sampling process of the NHDS database.

\section{RESULTS}

\section{Characteristics of the Entire Cohort}

An estimated 162 million adult patients were hospitalized in the United States from January 1, 2005 , through December 31, 2009, and 8.03 million (5\%) had a diagnosis of CKD. The majority of CKD patients (5.17 million [64.4\%]) were 65 years of age or older. Their median (minimum, maximum) age was $71(18,99)$ years, and 4.17 million patients $(51.9 \%)$ were men. Caucasian race was the most representative (4.16 million [65.8\%]), followed by African American (1.77 million [28.1\%]). The majority of hospital admissions were urgent/emergent (6.53 million [85.2\%]). Unspecified CKD was the most common CKD diagnosis (3.21 million patients 


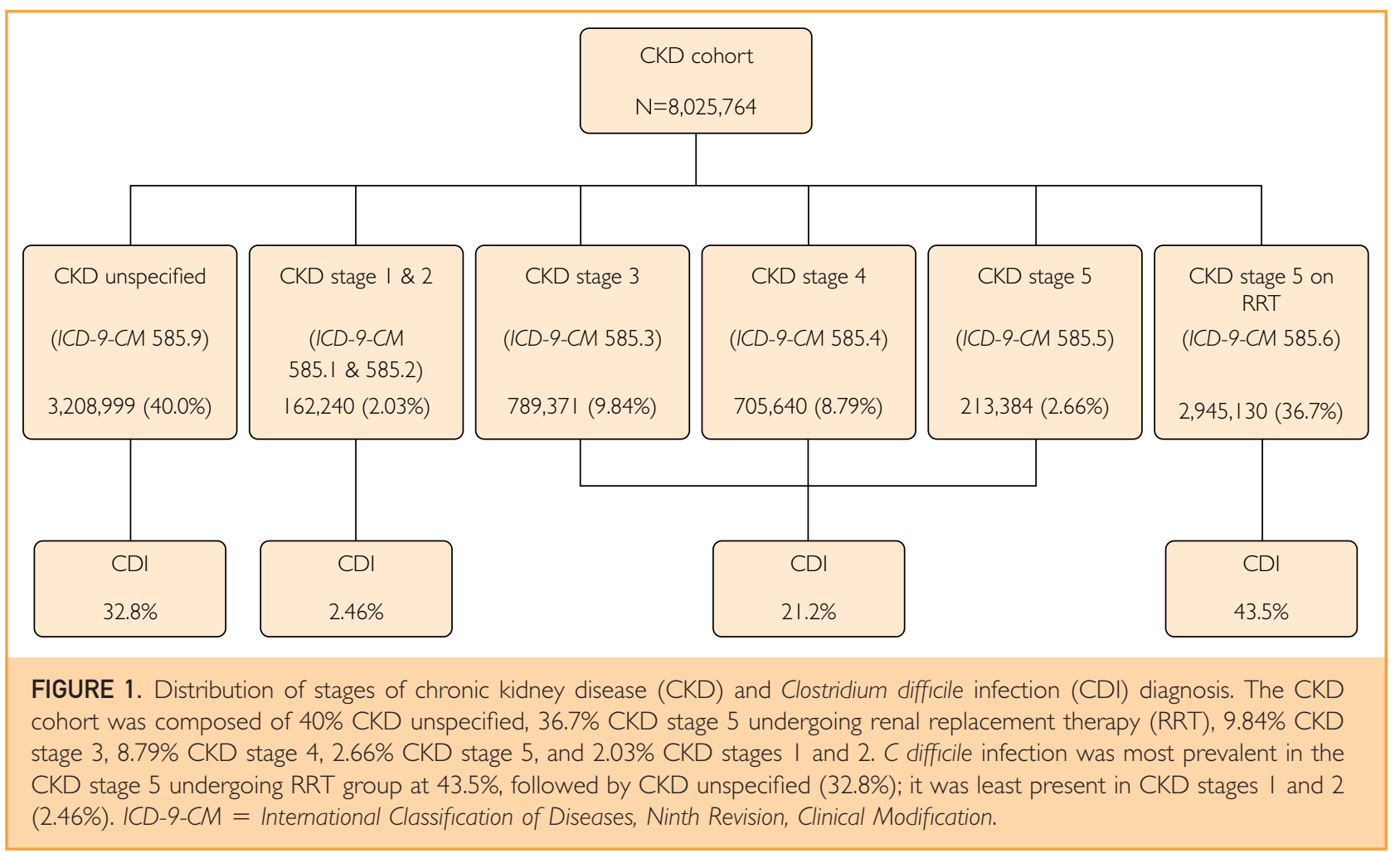

[40.0\%]), followed by CKD-5 undergoing dialysis (2.95 million patients [36.7\%]) (Figure 1).

A diagnosis of AKI was present in 1.55 million patients with CKD (19.3\%) and was most common among patients with unspecified CKD (0.77 million patients [48.3\%]). A total of 2.21 million patients

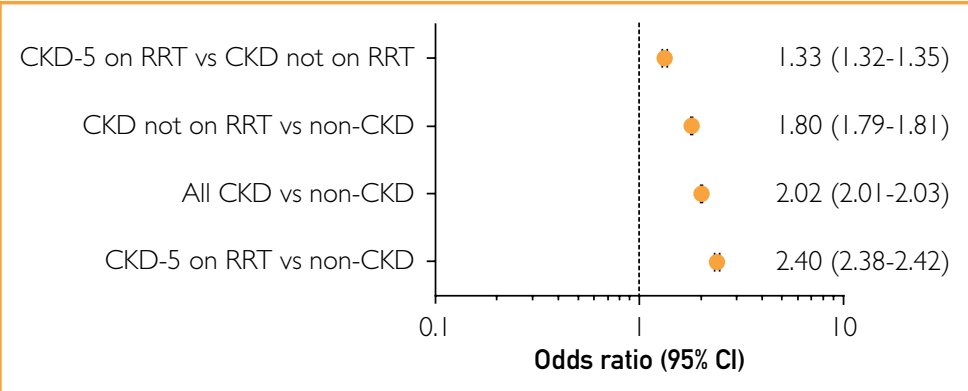

FIGURE 2. The odds ratio (OR) of Clostridium difficile infection (CDI) in patients with chronic kidney disease (CKD) not undergoing renal replacement therapy (RRT), those with stage 5 CKD (CKD-5) undergoing RRT, and non-CKD patients. All CKD patients had increased odds of having CDI compared with non-CKD patients (OR, 2.02). Patients with CKD-5 undergoing RRT had the greatest odds of having $\mathrm{CDI}$ compared with non-CKD patients (OR, 2.40). Among patients with CKD, those with stage 5 undergoing RRT had a 1.33 -fold increased odds of CDI compared with CKD patients not undergoing RRT.
(27.5\%) required renal replacement therapy during their hospitalization, 2.06 million (93.2\%) of whom were patients with CKD-5 undergoing long-term dialysis. Of the 1.55 million patients with AKI, 0.26 million $(10.3 \%)$ required renal replacement therapy.

\section{Rate in Patients With and Without CKD}

Patients with CKD were twice as likely to develop CDI as non-CKD patients (OR, 2.02; 95\% CI, 2.012.03; $P<.001$ ) (Figure 2). In addition, patients with CKD-5 undergoing long-term dialysis were at highest risk of CDI as compared with non-CKD patients (OR, 2.40; 95\% CI, 2.38-2.42; P<.001). Similarly, CKD patients who were not undergoing dialysis also had a higher rate of CDI than non-CKD patients (OR, 1.80; 95\% CI, 1.79-1.81; P<.001). Moreover, CKD patients who were undergoing long-term dialysis were more likely to acquire CDI than were CKD patients who were not undergoing dialysis (OR, 1.33; 95\% CI, 1.32-1.35; P<.001).

\section{Characteristics and Outcomes of CKD Patients With and Without CDI}

Patients with CKD who had CDI were older (median age, 75 [21, 97] years vs 71 [18, 99] years; $P<.001)$ and more likely to have had urgent/emergent (vs elective) admission (OR, 1.64; 95\% CI, 1.60-1.67; $P<.001$ ) compared with those without 


\begin{tabular}{|c|c|c|c|c|}
\hline Characteristic & $\begin{array}{c}\text { All CKD } \\
\left(\mathrm{N}=8.03 \times 10^{6}\right)\end{array}$ & $\begin{array}{l}\text { CKD without CDI } \\
\left(n=7.91 \times 10^{6}\right)\end{array}$ & $\begin{array}{c}\text { CKD with CDI } \\
\left(n=0.119 \times 10^{6}\right)\end{array}$ & $P$ value \\
\hline Age (y) & $71(18,99)$ & $71(18,99)$ & $75(21,97)^{c}$ & $<.001$ \\
\hline Male & $4.17 \times 10^{6}(51.9)$ & $4.10 \times 10^{6}(51.9)$ & $0.065 \times 10^{6}(54.5)^{d}$ & $<.001$ \\
\hline Race $^{e}$ & & & & $<.001$ \\
\hline Caucasian & $4.16 \times 10^{6}(65.8)$ & $4.09 \times 10^{6}(65.6)$ & $0.0735 \times 10^{6}(76.2)$ & \\
\hline African American & $1.77 \times 10^{6}(28.1)$ & $1.76 \times 10^{6}(28.2)$ & $0.0170 \times 10^{6}(17.6)$ & \\
\hline Asian & $0.121 \times 10^{6}(1.91)$ & $0.119 \times 10^{6}(1.92)$ & $0.00153 \times 10^{6}(1.59)$ & \\
\hline Other & $0.269 \times 10^{6}(4.25)$ & $0.265 \times 10^{6}(4.25)$ & $0.00447 \times 10^{6}(4.64)$ & \\
\hline Admission type $^{f}$ & & & & $<.001$ \\
\hline Routine & $1.13 \times 10^{6}(14.8)$ & $1.12 \times 10^{6}(14.9)$ & $0.011 \times 10^{6}(9.66)$ & \\
\hline Urgent/emergent & $6.53 \times 10^{6}(85.2)$ & $6.42 \times 10^{6}(85.1)$ & $0.104 \times 10^{6}(90.3)^{\mathrm{e}}$ & \\
\hline 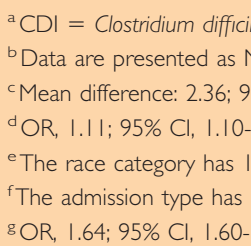 & $\begin{array}{l}\text { ction; } \mathrm{Cl}=\text { confidence } \\
\text { ercentage) or median ( } \\
\text { I, I.32-3.4I. } \\
\text { missing data; the denon } \\
\text { missing data; the denom }\end{array}$ & $\begin{array}{l}r \text { is } 6.33 \text { million. } \\
r \text { is } 7.66 \text { million. }\end{array}$ & sease; OR = odds ratio. & \\
\hline
\end{tabular}

CDI (Table 1). Moreover, CKD patients with CDI had significantly longer median hospital stay ( 7 days vs 4 days; $P<.001$ ) compared with those without $\mathrm{CDI}$ and were more likely to undergo colectomy and be dismissed to a health care facility (Table 2). Of concern, all-cause in-hospital mortality was significantly higher among CKD patients with CDI than those without CDI (6.62\% vs 3.99\%; P<.001). After adjustment for sex and clinically important comorbid conditions (cardiovascular disease; diabetes and its complications and hypertension-associated complications; liver disease; and obesity), CDI associations with increased colectomy rate, prolonged length of hospital stay, increased rate of discharge to a health care facility, and in-hospital mortality remained statistically significant (all $P<.001)$ (Table 2).

\section{Outcomes of CKD Patients With CDI With and Without Concomitant AKI}

As shown in Table 3, CKD patients with CDI and AKI had a significantly longer length of stay (median, 10 vs 7 days; $P=.0033$ ) than CKD patients with CDI but no AKI, higher risk of requiring colectomy and dismissal to a health care facility, and significantly worse in-hospital mortality $(10.8 \%$ vs

\begin{tabular}{|c|c|c|c|c|c|}
\hline Clinical outcome & $\begin{array}{l}\text { CKD without CDI } \\
\left(n=7.9 \mid \times 10^{6}\right)\end{array}$ & $\begin{array}{l}\text { CKD with CDI } \\
\left(n=0.119 \times 10^{6}\right)\end{array}$ & OR $(95 \% \mathrm{Cl})$ & $\begin{array}{c}\text { Adjusted OR } \\
(95 \% \mathrm{Cl})\end{array}$ & $P$ value \\
\hline Length of stay (d) & $4(1,381)$ & $7(1,68)$ & $\begin{array}{l}\text { Mean difference: } \\
3.89 \text { (3.39-4.39) }\end{array}$ & $\begin{array}{c}\text { Parameter estimate: } \\
\qquad 1.89\end{array}$ & $<.001$ \\
\hline $\begin{array}{l}\text { Discharge to health } \\
\text { care facility }{ }^{d}\end{array}$ & $18.3 \times 10^{5}(26.5)$ & $0.444 \times 10^{5}(44.4)$ & $2.21(2.18-2.23)$ & $2.22(2.19-2.25)$ & $<.001$ \\
\hline Colectomy & $2.20 \times 10^{4}(0.28)$ & $796(0.67)$ & $2.40(2.24-2.58)$ & $2.25(2.10-2.42)$ & $<.001$ \\
\hline In-hospital mortality & $3.16 \times 10^{5}(3.99)$ & $0.0791 \times 10^{5}(6.62)$ & $1.70(1.67-1.74)$ & $1.61(1.58-1.65)$ & $<.001$ \\
\hline \multicolumn{6}{|c|}{$\begin{array}{l}{ }^{a} \mathrm{CDI}=\text { Clostridium difficile infection; } \mathrm{Cl}=\text { confidence interval; } \mathrm{CKD}=\text { chronic kidney disease; } \mathrm{OR}=\text { odds ratio. } \\
\mathrm{b} \text { Data are presented as number (percentage) or median (minimum, maximum). } \\
{ }^{c} \text { P value of the adjusted OR. Adjustment was made for sex, diagnosis of cardiovascular disease (congestive heart failure, valvular disease, } \\
\text { and pulmonary and circulatory disease), peripheral vascular disease, diabetes and its complications, hypertension-associated complica- } \\
\text { tions, liver disease, and obesity for all the outcomes of interest. }\end{array}$} \\
\hline
\end{tabular}




\begin{tabular}{|c|c|c|c|c|c|}
\hline Clinical outcome & $\begin{array}{c}\text { AKI } \\
\left(n=2.98 \times 10^{4}\right)\end{array}$ & $\begin{array}{c}\text { No AKI } \\
\left(n=8.96 \times 10^{4}\right)\end{array}$ & OR $(95 \% \mathrm{Cl})$ & $\begin{array}{l}\text { Adjusted OR } \\
(95 \% \mathrm{Cl})\end{array}$ & $P$ value $^{c}$ \\
\hline Length of stay (d) & $10(1,60)$ & $7(1,68)$ & $\begin{array}{l}\text { Mean difference: } \\
2.34(0.101-4.57)\end{array}$ & $\begin{array}{c}\text { Parameter estimate: } \\
0.986\end{array}$ & .0084 \\
\hline $\begin{array}{l}\text { Discharge to health } \\
\text { care facility }\end{array}$ & $1.14 \times 10^{4}(48.2)$ & $3.30 \times 10^{4}(43.2)$ & $1.47(1.40-1.55)$ & $1.11(1.07-1.14)$ & $<.001$ \\
\hline Colectomy & $47 \mid(1.58)$ & $325(0.36)$ & $4.41(3.82-5.08)$ & $2.17(1.87-2.5 \mathrm{I})$ & $<.001$ \\
\hline In-hospital mortality & $3219(10.8)$ & $4688(5.23)$ & $2.19(2.09-2.30)$ & $2.18(2.07-2.29)$ & $<.001$ \\
\hline \multicolumn{6}{|c|}{$\begin{array}{l}{ }^{a} \mathrm{AKI}=\text { acute kidney injury; } \mathrm{CDI}=\text { Clostridium difficile infection; } \mathrm{Cl}=\text { confidence interval; } \mathrm{CKD}=\text { chronic kidney disease; } \mathrm{OR}=\text { odds } \\
\text { ratio. } \\
\text { b Data are presented as No. (percentage) or median (minimum, maximum). } \\
\text { c } P \text { value of the adjusted OR. Adjustment was made for sex, diagnosis of cardiovascular disease (congestive heart failure, valvular disease, } \\
\text { and pulmonary and circulatory disease), peripheral vascular disease, diabetes and its complications, hypertension-associated complica- } \\
\text { tions, liver disease, and obesity for discharge to care facility. For colectomy rate, peripheral vascular disease, obesity, diabetes and its } \\
\text { complications, and liver disease were removed from the model due to missing data. For in-hospital mortality, peripheral vascular disease } \\
\text { and obesity were removed from the model. } \\
\text { dExcluding patients who left against medical advice or died in the hospital. }\end{array}$} \\
\hline
\end{tabular}

5.23\%; $P<.001)$. These associations remained significant after multivariate adjustment (Table 3 ).

\section{Outcomes of CKD Patients With CDI Undergoing Long-term Dialysis}

Of the 8.03 million CKD patients, 2.95 million (36.7\%) had a diagnosis of CKD-5 undergoing dialysis. During the hospitalization, 2.06 million (93.2\%) of those with CKD-5 undergoing dialysis and 0.149 million $(6.77 \%)$ of all other CKD patients required renal replacement therapy. To examine the effect of long-term dialysis on CDI-associated outcomes, we first excluded the 0.149 million patients $(6.77 \%$ ) with other CKD diagnoses who required renal replacement therapy and analyzed clinical outcomes of CKD patients with CDI who did not undergo long-term dialysis and compared them with those in patients with CKD-5 and CDI who did undergo long-term dialysis. As shown in Table 4, on weighted univariate analysis, hospitalized patients with CKD and CDI who underwent long-term dialysis were less likely to require colectomy and be dismissed to a health care facility and had a significantly lower mortality rate (all $P<.001$ ) compared with patients who had CKD and CDI who did not undergo long-term dialysis. Similarly, after adjustment for sex and common comorbid conditions (logistic regression), CKD-5 patients with CDI who underwent long-term dialysis did not have an increased risk of adverse hospital-associated outcomes, although the magnitude of the difference between the groups diminished. There was no statistically significant difference in length of stay between the 2 groups in the adjusted model (Table 4).

\section{DISCUSSION}

Using a nationwide, hospital-based database (NHDS), we demonstrated that the rate of CDI is markedly increased in patients with CKD and that CDI is associated with significant hospital-associated morbidity and mortality. Chronic kidney disease patients with CDI had a longer hospital stay, an increased rate of colectomy during the hospitalization, a higher likelihood of dismissal to a health care facility, and a significant increase in all-cause in-hospital mortality, even after adjusting for known comorbid conditions. Thus, our results suggest that CKD is a significant risk factor for both CDI occurrence and worse hospital-associated outcomes.

Previous studies evaluating the rate of CDI in patients with CKD have demonstrated inconsistent results. Although some have shown that the risk of CDI is higher in dialysis patients but not in nondialysis CKD patients, ${ }^{14,15}$ at least one investigation has found that CDI was almost 4 times higher among patients with renal disease (both acute and chronic) in a nephrology unit at a tertiary hospital as compared with patients in the remainder of the hospital. ${ }^{13}$ The results from surveying the NHDS database showed a significantly higher rate of CDI in both dialysis and nondialysis CKD patients as compared with patients without CKD. Therefore, it appears that patients with CKD have a unique predilection to acquire CDI and that patients undergoing dialysis are at particularly higher risk of CDI than are CKD patients not undergoing dialysis. However, because CKD stages in our study were based on ICD9-CM codes, the impact of varying degrees of eGFR impairment on the occurrence of CDI could not be adequately assessed. The higher rate of CDI in pa- 


\begin{tabular}{|c|c|c|c|c|c|}
\hline Clinical outcome & $\begin{array}{l}\text { CKD-5 on long-term } \\
\text { dialysis }(n=43,796)\end{array}$ & $\begin{array}{l}\text { CKD, no dialysis } \\
(n=52,638)\end{array}$ & OR $(95 \% \mathrm{Cl})$ & $\begin{array}{l}\text { Adjusted OR } \\
(95 \% \mathrm{Cl})\end{array}$ & $P$ value ${ }^{c}$ \\
\hline Length of stay (d) & $8(1,68)$ & $7(1,62)$ & $\begin{array}{c}\text { Mean difference: } \\
0.354(-0.889 \text { to } 1.60)\end{array}$ & $\begin{array}{l}\text { Parameter estimate: } \\
0.344\end{array}$ & .304 \\
\hline Discharge to health care facility ${ }^{d}$ & $1.79 \times 10^{4}(40.9)$ & $2.39 \times 10^{4}(45.5)$ & $0.830(0.809-0.852)$ & $0.96 \mid(0.935-0.989)$ & .0063. \\
\hline Colectomy & $93(0.18)$ & $703(1.11)$ & $0.160(0.129-0.199)$ & $0.327(0.260-0.405)$ & $<.001$ \\
\hline In-hospital mortality & $0.325 \times 10^{4}(6.26)$ & $0.460 \times 10^{4}(7.24)$ & $0.856(0.817-0.896)$ & $0.921(0.875-0.968)$ & .0013 \\
\hline \multicolumn{6}{|c|}{$\begin{array}{l}\text { a } \mathrm{CDI}=\text { Clostridium difficile infection; } \mathrm{Cl}=\text { confidence interval; } \mathrm{CKD}=\text { chronic kidney disease; } \mathrm{OR}=\text { odds ratio. } \\
{ }^{\mathrm{b}} \text { Data are presented as No. (percentage) or median (minimum, maximum). } \\
\text { cP value of the adjusted OR. Adjustment was made for sex, diagnosis of cardiovascular disease (congestive heart failure, valvular disease, and pulmonary and circulatory } \\
\text { disease), peripheral vascular disease, diabetes and its complications, hypertension-associated complications, liver disease, and obesity for length of stay and discharge to } \\
\text { health care facility. For colectomy, only sex, cardiovascular disease, and hypertension-associated complications were included in the model. For in-hospital mortality, } \\
\text { adjustment was made for all variables except obesity and peripheral vascular disease. } \\
\text { dExcluding patients who left against medical advice or died in the hospital. }\end{array}$} \\
\hline
\end{tabular}

tients with CKD may be explained by several risk factors. In addition to well-established risk factors associated with the development of CDI, such as older age and prolonged hospitalization, which were demonstrated in the current study cohort with CDI, other unique risk factors in CKD may predispose patients to CDI. These include gastric acid suppression, ${ }^{17}$ increased colonization with Clostridium species ${ }^{18}$ impaired immune system function, ${ }^{11,19}$ and increased antibiotic use. ${ }^{14}$ We speculate that these unique risk factors could account, at least in part, for the increased rate of CDI in CKD patients. Due to the limitations of the NHDS, we were unable to examine CDI risk factors and treatment for CDI.

Similar to our results, previous studies with smaller numbers of CKD patients have shown a 3-fold increase in mortality associated with CDI. ${ }^{15,20}$ Our results showed that after adjusting for common comorbid conditions, CDI was associated with a significantly higher risk of in-hospital mortality in all CKD patients. Because CKD and CDI are both associated with increased morbidity and mortality, the association of increased mortality in CKD patients with CDI appears consistent. Not surprisingly, CKD patients with CDI who had concomitant AKI had significantly worse outcomes, which is consistent with the known adverse impact of AKI in hospitalized patients with or without CKD. ${ }^{21-24}$ It is important to note that the increased rate of colectomy among CKD patients with AKI and CDI in our study likely represented a more complicated CDI course. This is consistent with the Infectious Diseases Society of America recommendation that the presence of any AKI in the course of CDI is a marker of CDI severity. ${ }^{2}$

The demonstration that among all CKD patients, those with CKD-5 undergoing long-term di- alysis who developed CDI were less likely to suffer adverse outcomes as compared with CKD patients not undergoing dialysis was unexpected. Patients with CKD who were undergoing long-term dialysis were less likely to require colectomy and be dismissed to a health care facility, and surprisingly, they had lower in-hospital mortality. We postulate that the lower rate of colectomy could be due to a higher threshold for surgery in long-term dialysis patients in general. We speculate that the association of lower mortality in CKD-5 patients with CDI undergoing long-term dialysis could be due to more timely admissions, regular nephrology care while in the hospital, or more extensive monitoring in CKD-5 patients undergoing dialysis. It is noteworthy that the large sample size and narrow CIs with relatively small ORs in that analysis of CKD-5 on chronic dialysis could implicate a statistical significance without clear clinical relevance.

Our study has several limitations that warrant discussion. First, although our study included the entire hospitalized population in the NHDS database, the national survey represents approximately $1 \%$ of all hospitalizations in the United States and therefore tends to underestimate disease prevalence. However, weighted analysis allows for an estimation of nationwide disease prevalence. In addition, the database uniquely identifies each dismissal, but there is a possibility that patients may be represented by more than one dismissal record. In addition, we were unable to obtain information on serum creatinine concentration, proteinuria, and eGFR to confirm CKD staging. This may explain why CKD in our cohort only represented $5 \%$ of the entire hospitalized population included in the NHDS. Therefore, CKD patients were likely underrepresented in this database. However, despite the underestimation in $\mathrm{CKD}$, the dialysis population 
constituted a large proportion of the hospitalized CKD patients in this database, which is consistent with previous observations, ${ }^{25,26}$ and we were able to show significant differences in CDI rate as well as complications in CKD patients.

The limitations of CKD estimation using ICD9-CM codes have been previously recognized and lead to underestimation of CKD prevalence and variable sensitivity. ${ }^{27}$ However, previous validation studies have shown that the specificity of CKD-3, CKD-4, and CKD-5 was as high as $95 \%{ }^{28}$ and that accounting for dialysis use tends to improve sensitivity. We attempted to compensate for these limitations by combining all CKD stages. Moreover, the diagnosis code for CKD-5 requiring renal replacement therapy is unique, and we were able to compare CKD patients undergoing dialysis with those not undergoing dialysis with confidence.

Second, we lacked information on potential confounders, such as CDI colonization vs true infection, antibiotic exposure, CDI treatment, $C$ difficile strain, nosocomial vs community-acquired CDI, and important laboratory parameters. The diagnosis of CDI was made by ICD-9-CM code rather than by clinical symptoms and positive stool assay results. Nevertheless, the ICD-9-CM code for CDI has been shown to correlate well with the results of $C$ difficile toxin assay. ${ }^{29}$ The diagnosis of CDI as measured by the NHDS database was similar to the rate of CDI as measured by toxin assay in another study. ${ }^{30}$ Third, the comorbid conditions used for adjustment lack precision and therefore may have impacted the findings. Lastly, this study by design provides data that support a strong association between CDI and CKD but not a causal relationship.

\section{CONCLUSION}

Our investigation represents the largest study to date to examine CDI in CKD patients and demonstrated troubling results. Our findings suggest that CKD patients have a higher rate of CDI, prolonged hospital stay, and colectomy and are more likely to be dismissed to a health care facility compared with CKD patients without CDI. However, based on our results, CKD patients with CDI who undergo longterm dialysis are not at significantly higher risk of adverse hospital-associated outcomes. Future prospective studies are needed to confirm these findings, to better characterize the impact of CDI in the CKD population, and to identify effective CDI prevention in CKD patients, who appear to have an increased risk of CDI acquisition.

Abbreviations and Acronyms: $\mathrm{AKI}=$ acute kidney injury; CDI = Clostridium difficile infection; $\mathbf{C l}=$ confidence interval; CKD = chronic kidney disease; eGFR = estimated glomerular filtration rate; $I C D-9-C M=$ International Classification of
Diseases, Ninth Revision, Clinical Modification; NHDS $=\mathrm{Na}$ tional Hospital Discharge Survey; OR = odds ratio

Correspondence: Address to Qi Qian, MD, Division of Gastroenterology and Hepatology, Mayo Clinic, 200 First St SW, Rochester, MN 55905 (qian.qi@mayo.edu).

\section{REFERENCES}

1. Khanna S, Pardi DS, Aronson SL, et al. The epidemiology of community-acquired Clostridium difficile infection: a population-based study. Am J Gastroenterol. 2012;107( I ):89-95.

2. Cohen DN, Gerding SH, Johnson S, et al; Society for Healthcare Epidemiology of America; Infectious Diseases Society of America. Clinical practice guidelines for Clostridium difficile infection in adults: 2010 update by the Society for Healthcare Epidemiology of America (SHEA) and the Infectious Diseases Society of America (IDSA). Infect Control Hosp Epidemiol. 2010;3। (5):431-455.

3. McDonald LC, Owings M, Jernigan DB. Clostridium difficile infection in patients discharged from US short-stay hospitals, 1996-2003. Emerg Infect Dis. 2006; 12(3):409-415.

4. Khanna S, Pardi DS. The growing incidence and severity of Clostridium difficile infection in inpatient and outpatient settings. Expert Rev Gastroenterol Hepatol. 20 I0;4(4):409-4I 6.

5. Khanna S, Pardi DS, Aronson SL, Kammer PP, Baddour LM. Outcomes in community-acquired Clostridium difficile infection. Aliment Pharmacol Ther. 2012;35(5):613-618.

6. Redelings MD, Sorvillo F, Mascola L. Increase in Clostridium difficile-related mortality rates, United States, 1999-2004. Emerg Infect Dis. 2007;13(9):1417-1419.

7. Pepin J, Alary ME, Valiquette $L$, et al. Increasing risk of relapse after treatment of Clostridium difficile colitis in Quebec, Canada. Clin Infect Dis. 2005;40( I I): I 59 I - 1597.

8. Pépin J, Valiquette L, Alary ME, et al. Clostridium difficile-associated diarrhea in a region of Quebec from 1991 to 2003: a changing pattern of disease severity. CMAJ. 2004; I7I (5):466472.

9. Morrison RH, Hall NS, Said M, et al. Risk factors associated with complications and mortality in patients with Clostridium difficile infection. Clin Infect Dis. 201 1;53(12):1 173-1 178.

10. Dial S, Alrasadi K, Manoukian C, Huang A, Menzies D. Risk of Clostridium difficile diarrhea among hospital inpatients prescribed proton pump inhibitors: cohort and case-control studies. CMAJ. 2004; I 7 I ( I):33-38.

11. Anand A, Glatt AE. Clostridium difficile infection associated with antineoplastic chemotherapy: a review. Clin Infect Dis. 1993; 17(I): 109-113.

12. McFarland LV, Mulligan ME, Kwok RY, Stamm WE. Nosocomial acquisition of Clostridium difficile infection. N Engl J Med. 1989;320(4):204-210

13. Cunney RJ, Magee C, McNamara E, Smyth EG, Walshe J. Clostridium difficile colitis associated with chronic renal failure. Nephrol Dial Transplant. 1998; 13(I I):2842-2846.

14. Eddi R, Malik MN, Shakov R, Baddoura WJ, Chandran C, Debari VA. Chronic kidney disease as a risk factor for Clostridium difficile infection. Nephrology (Carlton). 20 I0;15(4):47I 475.

15. Yousuf K, Saklayen MG, Markert RJ, Barde C), Gopalswamy N Clostridium difficile-associated diarrhea and chronic renal insufficiency. South Med J. 2002;95(7):681-683.

16. US Renal Data System. USRDS 2000 Annual Data Report: Atlas of Chronic Kidney Disease and End-Stage Renal Disease in the United States. Bethesda, MD: 2000. 
17. McConnell JB, Stewart WK, Thjodleifsson B, Wormsley KG Gastric function in chronic renal failure: effects of maintenance haemodialysis. Lancet. 1975;2(7945): I I 2 1 - I 123.

18. Aronsson B, Barany P, Nord CE, Nyström B, Stenvinkel P. Clostridium difficile-associated diarrhoea in uremic patients. Eur J Clin Microbiol. 1987;6(3):352-356.

19. Pardi DS. Recurrent Clostridium difficile infection: an immunodeficiency state? Clin Gastroenterol Hepatol. 2007;5(6):672673.

20. Bárány P, Stenvinkel P, Nord CE, Bergström J. Clostridium difficile infection - a poor prognostic sign in uremic patients? Clin Nephrol. 1992;38( I):53-57.

21. Coca SG, Peixoto AJ, Garg AX, Krumholz HM, Parikh CR. The prognostic importance of a small acute decrement in kidney function in hospitalized patients: a systematic review and meta-analysis. Am J Kidney Dis. 2007;50(5):712-720.

22. Coca SG, Yusuf B, Shlipak MG, Garg AX, Parikh CR. Longterm risk of mortality and other adverse outcomes after acute kidney injury: a systematic review and meta-analysis. Am Kidney Dis. 2009;53(6):961-973.

23. Hsu CY, Chertow GM, McCulloch CE, Fan D, Ordoñez JD, Go AS Nonrecovery of kidney function and death after acute on chronic renal failure. Clin J Am Soc Nephrol. 2009;4(5):891-898.
24. Pannu N, James M, Hemmelgarn BR, Dong J, Tonelli M, Klarenbach S; Alberta Kidney Disease Network. Modification of outcomes after acute kidney injury by the presence of CKD. Am J Kidney Dis. 2011;58(2):206-213.

25. Khan SS, Kazmi WH, Abichandani R, Tighiouart H, Pereira BJ Kausz AT. Health care utilization among patients with chronic kidney disease. Kidney Int. 2002;62(1):229-236.

26. Arora P, Kausz AT, Obrador GT, et al. Hospital utilization among chronic dialysis patients. J Am Soc Nephrol. 2000; I I (4): 740-746

27. Vlasschaert ME, Bejaimal SA, Hackam DG, et al. Validity of administrative database coding for kidney disease: a systematic review. Am J Kidney Dis. 201 1;57(1):29-43.

28. Kern EF, Maney M, Miller DR, et al. Failure of ICD-9-CM codes to identify patients with comorbid chronic kidney disease in diabetes. Health Serv Res. 2006;4I (2):564-580.

29. Dubberke ER, Reske KA, McDonald LC, Fraser VJ. ICD-9 codes and surveillance for Clostridium difficile-associated disease. Emerg Infect Dis. 2006; I 2(10): I576- 1579.

30. Dubberke ER, Butler AM, Nyazee HA, et al; Centers for Disease Control and Prevention Epicenters Program. The impact of ICD-9-CM code rank order on the estimated prevalence of Clostridium difficile infections. Clin Infect Dis. 2011; 53(I):20-25 